\title{
Gene expression profiles and protein-protein interaction networks during tongue carcinogenesis in the tumor microenvironment
}

\author{
WEI SUN ${ }^{1,2^{*}}$, ZETING QIU ${ }^{1,2^{*}}$, WENQI HUANG ${ }^{1,2}$ and MINGHUI CAO ${ }^{1,2}$ \\ ${ }^{1}$ Department of Anesthesiology, Sun Yat-sen Memorial Hospital, Sun Yat-sen University; ${ }^{2}$ Department of Anesthesiology, \\ The First Affiliated Hospital of Sun Yat-sen University, Guangzhou, Guangdong 510080, P.R. China
}

Received December 2, 2016; Accepted July 7, 2017

DOI: $10.3892 / \mathrm{mmr} .2017 .7843$

\begin{abstract}
Oral tongue squamous cell carcinoma (OTSCC) has a high incidence and is associated with a high mortality rate. Studies regarding the potential molecular mechanism of OTSCC in the tumor microenvironment (TME) are required. The present study aimed to perform bioinformatic analysis to identify important nodes, clusters and functional pathways during tongue carcinogenesis in the TME. After downloading the gene expression data of GSE42780, differentially expressed genes (DEGs) among carcinoma, dysplastic and normal samples in epithelia and fibroblasts were identified using the affy and limma packages with $\mathrm{R}$ version 3.3. Subsequently, the Database for Annotation, Visualization and Integrated Discovery was employed to perform Gene Ontology (GO) and Kyoto Encyclopedia of Genes and Genomes (KEGG) enrichment analysis. Furthermore, a protein-protein interaction (PPI) network was constructed by using the Search Tool for the Retrieval of Interacting Genes/Proteins and analyzed by Cytoscape software. In total, 85 DEGs were identified for tongue epithelia and 46 DEGs were identified for fibroblasts. Neutrophil chemotaxis and inflammatory response from $\mathrm{GO}$, and cytokine-cytokine receptor interaction from KEGG were enriched for epithelia and fibroblasts. The PPI network revealed that $\mathrm{C}-\mathrm{X}-\mathrm{C}$ motif chemokine ligand $(\mathrm{Cxcl}) 1, \mathrm{Cxcl10}$, Cxcl13, Cxcl2 and pro-platelet basic protein were a key cluster for epithelia, and interleukin (Il)1 $\beta$, Il1 receptor 2, Illa and Il1
\end{abstract}

Correspondence to: Professor Minghui Cao, Department of Anesthesiology, Sun Yat-sen Memorial Hospital, Sun Yat-sen University, 107 Yanjiang Road, Guangzhou, Guangdong 510080, P.R. China

E-mail: caominghui_sysu@163.com

Professor Wenqi Huang, Department of Anesthesiology, The First Affiliated Hospital of Sun Yat-sen University, 58 Zhongshan Second Road, Guangzhou, Guangdong 510080, P.R. China

E-mail: huangwenqi86@aliyun.com

*Contributed equally

Key words: gene expression profiles, protein-protein interaction, tongue cancer, tongue carcinoma, tumor microenvironment receptor antagonist were a key cluster for fibroblasts. Therefore, the results indicate that fibroblasts and cytokines associated with an inflammatory immune response contributed substantially to tongue carcinogenesis in the TME, which is useful for the development of OTSCC targeted therapy. However, further investigation is required to elucidate the molecular and cellular mechanisms underlying the inflammatory immune network in the TME.

\section{Introduction}

Head and neck squamous cell carcinoma (HNSCC) is the sixth leading type of cancer globally, of which oral cavity squamous cell carcinoma (OCSCC) accounts for $\sim 50 \%$ of cases (1). Etiologically, tobacco smoking and alcohol consumption are two of the major risk factors for OCSCC (2) Patients with OCSCC often present with local invasion and lymph node metastases, but usually without distant metastases. Oral tongue squamous cell carcinoma (OTSCC) is a form of OCSCC that originates from the anterior region of the tongue (3). In addition to tobacco and alcohol, infection with human papilloma virus (HPV), particularly HPV16, is strongly associated with the occurrence of tongue cancer (4). OCSCC, including OTSCC, is classified into stages I-IV according to the tumor, node and metastasis (TNM) staging system of the American Joint Committee on Cancer (AJCC) or the International Union for Cancer Control (5). For the AJCC stage I and II early OTSCC, the 5-year survival rates were reported to be 67 and $51 \%$, respectively, based on a population-based database termed Surveillance, Epidemiology and End Results (SEER) program (6). While, for stage III and IV advanced OTSCC, the 5-year disease-specific survival rates were 39 and 27\%, respectively (7). Oral carcinogenesis is a complex, diverse and successive process that includes normal, dysplasia and carcinoma stages, and the tumor microenvironment (TME) has an important role in malignant transformation $(8,9)$.

Microarrays based on gene expression are novel tools that are employed to understand the multiple interactions and identify the core networks of cancer (10) Publicly available microarray data and innovative bioinformatics tools have improved the ability to perform genome-wide expression studies independently. Various genetic changes have been identified by computational statistical methods in certain gene expression arrays involving $\operatorname{HNSCC}(11,12)$. However, 
identification of the key genes or pathways involved in the development of oral carcinogenesis is required, which will contribute to the discovery of core mechanical interactions and the development of targeted therapy for OTSCC.

It is hypothesized that the expression of certain genes is dysregulated during oral cancer carcinogenesis. Wu et al (13) investigated interleukin (Il) $1 \beta$ as the key gene in the TME during oral carcinogenesis via bioinformatic network construction, which was verified by immunohistochemical staining of human and rat samples. Subsequently, chemoprevention targeting at Il1 was confirmed to interrupt oral carcinogenesis in in vitro and in vivo experiments. However, in carcinogenesis, modules composed of functional genetic clusters usually cooperate together to fulfill specific functions, rather than a single key gene.

The present study performed gene expression profile analysis based on public microarray data from the Gene Expression Omnibus (GEO). By using GSE42780, the current study compared normal, dysplastic and carcinoma tissues in the TME. Differentially expressed genes (DEGs) were identified and key pathways of DEGs were enriched. In addition, a protein-protein interaction (PPI) network was constructed and analyzed to identify the potential hub modules of OTSCC.

\section{Materials and methods}

Microarray data. The gene expression data of the microarray GSE42780, based on the platform of GPL1355 (Affymetrix Rat Genome 230 2.0 Array), was downloaded from the GEO (www .ncbi.nlm.nih.gov/geo) of the National Center of Biotechnology Information. For GSE42780, following establishment of the 4-nitroquinoline oxide-induced tongue cancer model, RNA samples of rat tongue epithelia and submucosal fibroblasts were collected for gene microarray scanning, including 3 from normal control, 3 from dysplasia and 3 from carcinoma, as described by Wu et al (13).

Data processing. All of the data analysis in the present study was conducted by $\mathrm{R}$ statistical software version 3.3 (www.r-project.org/) along with an open source software for bioinformatics called Bioconductor (version 3.3; bioconductor.org/). Following robust multichip average background correcting, quantile normalizing and calculation of expression using the affy package (www.bioconductor .org/packages $/ 3.3 / \mathrm{bioc} / \mathrm{html} / \mathrm{affy} . \mathrm{html}$; version 1.50.0), thegene expression profile for all samples was obtained. Subsequently, a linear model was fitted and empirical Bayes statistics were computed by using the limma package (version 3.28.21; www .bioconductor.org/packages/3.3/bioc/html/limma.html). As a result, the significant DEGs among groups of normal control, dysplasia and carcinoma in tongue epithelia and submucosal fibroblasts samples were identified using a Venn diagram webtool (bioinformatics.psb.ugent.be/webtools/Venn/). Significant DEGs were selected with criteria of $\mathrm{P}<0.01$ and $\mid \log 2$ fold change $(\mathrm{FC}) \mid \geq 1.5$.

Gene Ontology (GO) enrichment and Kyoto Encyclopedia of Genes and Genomes (KEGG) pathway analysis. GO (http://geneontology.org) offers a biological model that
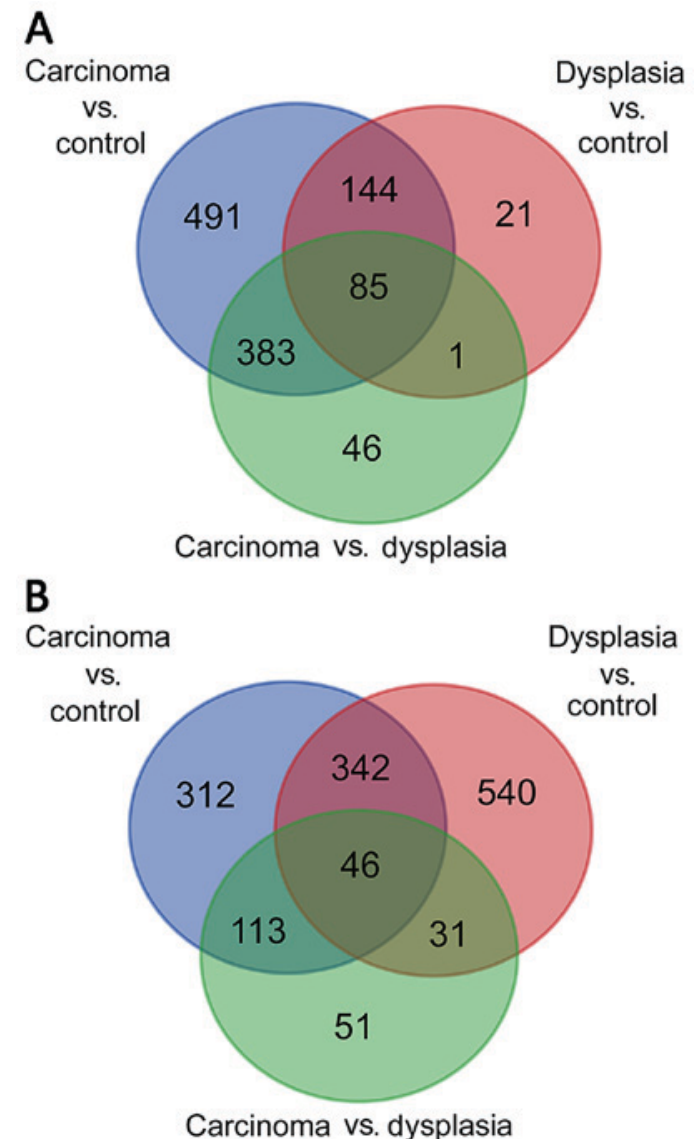

Figure 1. Venn diagrams of DEGs among carcinoma, dysplasia and control groups. DEGs among carcinoma, dysplasia and control groups in (A) epithelia and (B) fibroblasts. DEG, differentially expressed gene.

classifies gene functions into biological process, molecular function and cellular component (14). KEGG (www. genome/ad.jp/kegg/) is a database regarding genomes, biological pathways, diseases, drugs and chemical substances (15). The Database for Annotation, Visualization and Integrated Discovery (DAVID; https://david.ncifcrf.gov/), an integration of biological data and analysis tools, provides comprehensive functional annotation tools for a large number of genes (16). In the current study, GO annotation analysis and KEGG pathway enrichment analysis of DEGs was performed using DAVID tools online. $\mathrm{P}<0.05$ and gene counts $>2$ were considered indicate significance.

PPI construction. The Search Tool for the Retrieval of Interacting Genes/Proteins (STRING; string-db.org/) database provides a critical assessment and integration of PPI, including direct (physical) and indirect (functional) associations in a given organism (15). In the current study, the DEGs that were identified previously were uploaded to the STRING database to construct a PPI network, which provided improved comprehension of the physical and functional organization of the proteome systematically. Subsequently, the PPI network was reconstructed with Cytoscape software (version 3.3.0; www.cytoscape.org/). As nodes with a high degree of connectivity contribute more to the stability of the network, the connectivity degree of each protein node in the PPI network was calculated and the top 


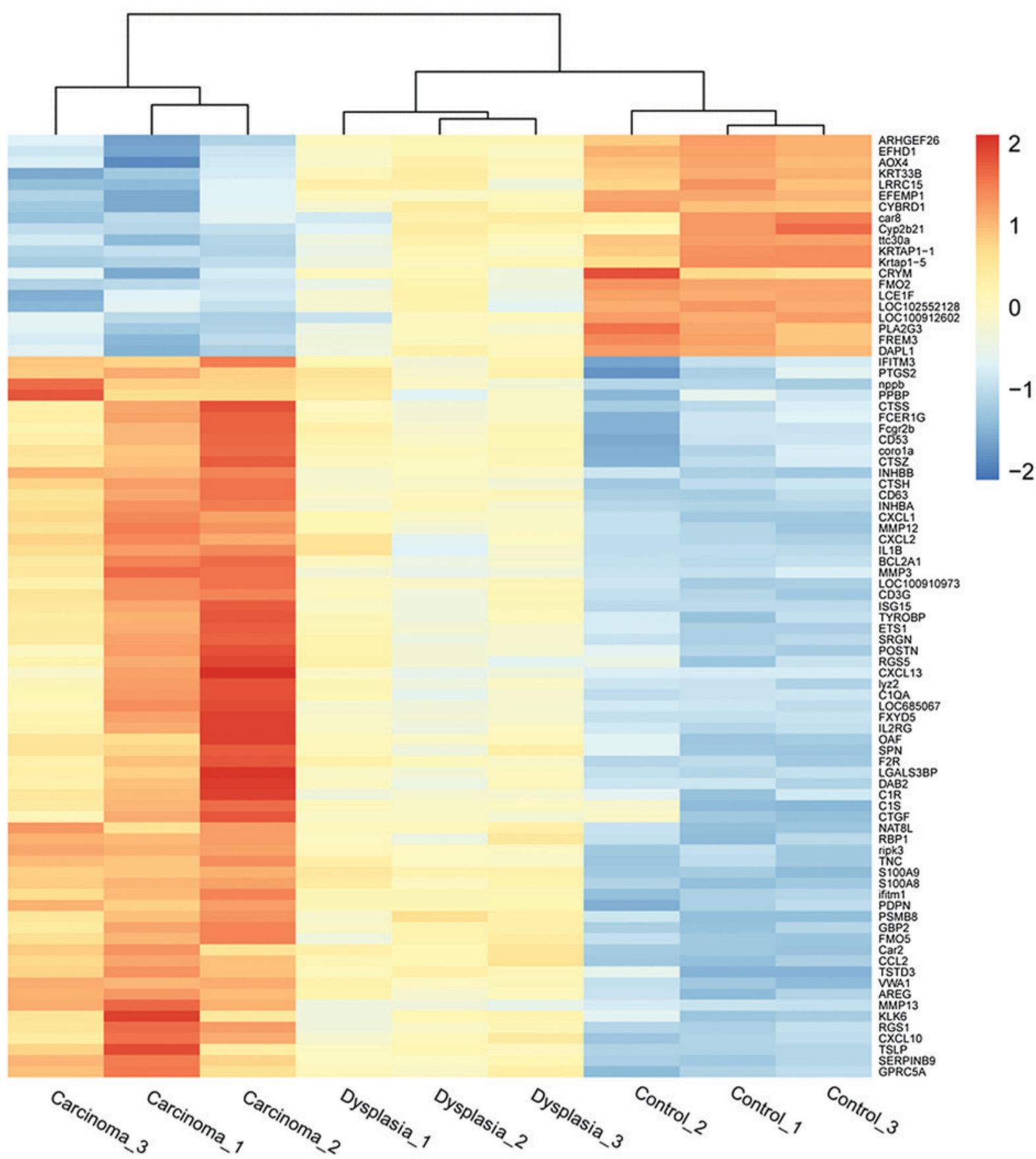

Figure 2. Heatmap of differentially expressed genes for epithelia. The degree of expression is indicated by different colors, with expression increasing between blue and orange. Blue, low expression; orange, high expression.

five were identified as the hub nodes using the Cytoscape plugin NetworkAnalyzer (release 2.7; med.bioinf.mpi-inf. mpg.de/netanalyzer/index.php). Finally, all significant genes were clustered into several groups to identify the important clusters using the Cytoscape plugin MCODE (version 1.4.2; apps.cytoscape.org/apps/mcode).

\section{Results}

$D E G$ analysis. For tongue epithelia samples, with the criteria of $\mathrm{P}<0.01$ and $|\log 2(\mathrm{FC})| \geq 1.5,1,103$ DEGs were identified when comparing carcinoma with normal control, 251 DEGs when comparing dysplasia with normal control and 515 DEGs when comparing carcinoma with dysplasia. As presented in Fig. 1A, following construction of a Venn diagram, 85 DEGs were significantly differentially expressed among all three groups.

Regarding submucosal fibroblast samples, 813 DEGs were identified when comparing carcinoma with normal control, 959 DEGs when comparing dysplasia with normal control and 241 DEGs when comparing carcinoma with dysplasia. As demonstrated in Fig. 1B, the Venn diagram identified 46 DEGs that were significantly differentially expressed among all three groups. Heatmaps indicating differential expression of genes among the three groups in epithelia and fibroblasts are presented in Figs. 2 and 3, respectively. 


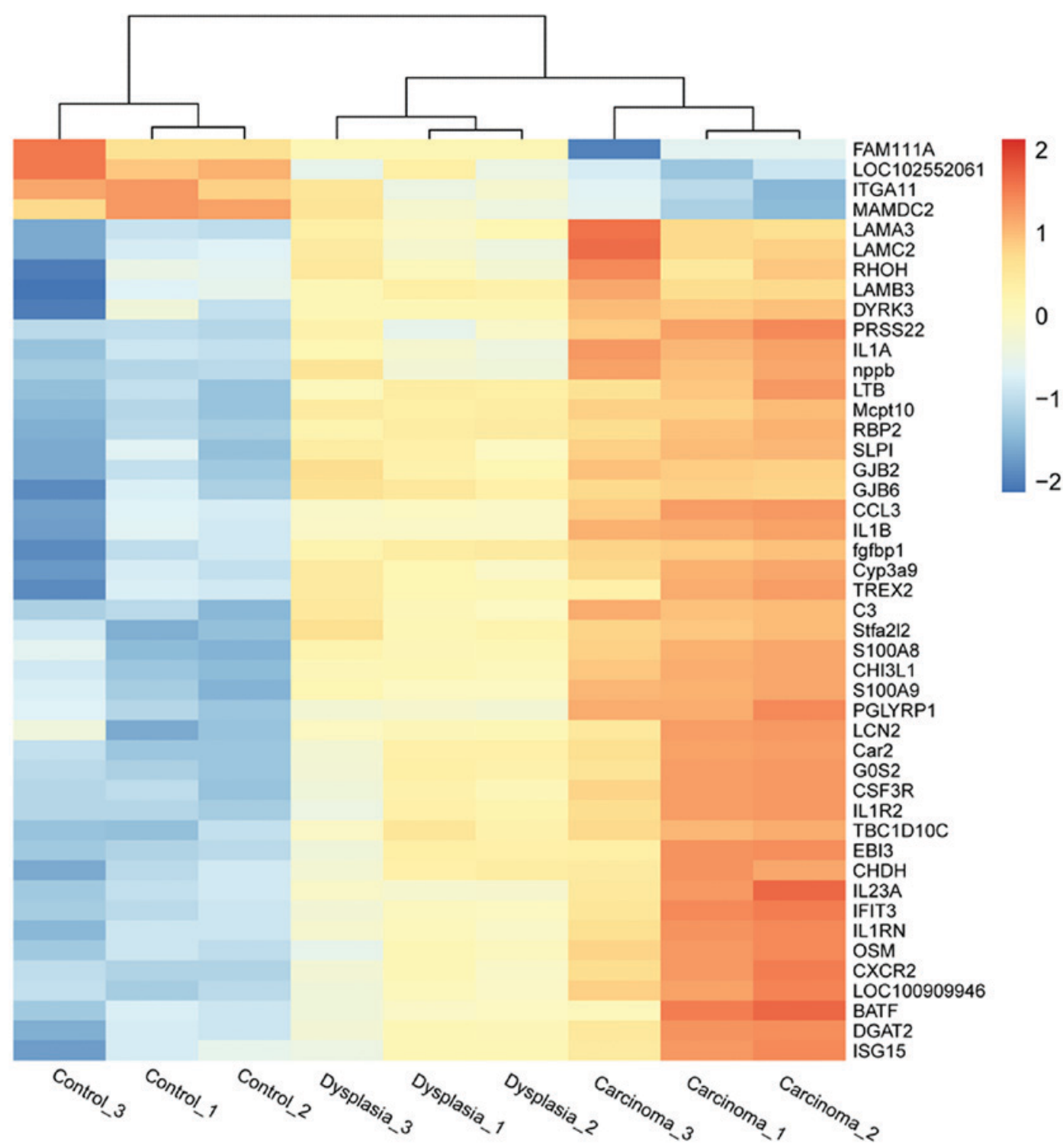

Figure 3. Heatmap of differentially expressed genes for fibroblasts. The degree of expression is indicated by different colors, with expression increasing between blue and orange. Blue, low expression; orange, high expression.

Functional enrichment analyses. For tongue epithelia samples, the top five significant GO biological process terms for DEGs were principally associated with neutrophil chemotaxis, response to lipopolysaccharide, inflammatory response, response to estradiol and positive regulation of cell migration, as demonstrated in Table I. The top five significant KEGG pathways of DEGs were enriched in the tumor necrosis factor (TNF) signaling pathway, cytokine-cytokine receptor interaction, Staphylococcus aureus infection, chemokine signaling pathway, and complement and coagulation cascades (Table II).

For submucosal fibroblast samples, the top five significant GO biological process terms of DEGs were principally associated with neutrophil chemotaxis, response to lipopolysaccharide, inflammatory response, cytokine-mediated signaling pathway and fever generation (Table III). The top five significant KEGG pathways of DEGs were enriched in cytokine-cytokine receptor interaction, rheumatoid arthritis, amoebiasis, pertussis and hematopoietic cell lineage (Table IV).
PPI analysis. For DEGs of tongue epithelia samples, 42 nodes and 75 edges were included in the PPI network based on the STRING database, as presented in Fig. 4A. Topological analysis by plugin NetworkAnalyzer identified Il1 $\beta, \mathrm{C}-\mathrm{C}$ motif chemokine ligand 2, matrix metallopeptidase 13, prostaglandin-endoperoxide synthase 2 and C-X-C motif chemokine ligand (Cxcl) 10 as hub nodes, which were considered to be key proteins in the whole network. A module consisting of Cxcl1, Cxcl10, Cxcl13, Cxcl2 and pro-platelet basic protein $(\mathrm{Ppbp})$ was recognized with a score $>4$ by plugin MCODE as a significant cluster in the PPI network. DEGs of this module were significantly associated with the TNF signaling pathway and cytokine-cytokine receptor interaction.

Regarding submucosal fibroblast samples, 22 nodes and 22 edges were included in the PPI network, as demonstrated in Fig. 4B. Topological analysis identified Il1 $\beta$, Il1 receptor (Il1r) 2, Il1 $\alpha$, Illr antagonist (I11rn) and Il23 $\alpha$ as key proteins. A module consisting of Il1 $\beta, \mathrm{Il} 1 \mathrm{r} 2$, Ill $\alpha$ and Il1rn was recognized 
Table I. GO biological process enrichment analyses of differentially expressed genes for epithelia.

\begin{tabular}{lrc}
\hline GO term & Count & P-value \\
\hline GO:0030593; neutrophil chemotaxis & 8 & $5.23 \times 10^{-9}$ \\
GO:0032496; response to & 12 & $2.92 \times 10^{-8}$ \\
lipopolysaccharide & & \\
GO:0006954; inflammatory response & 12 & $5.72 \times 10^{-8}$ \\
GO:0032355; response to estradiol & 10 & $1.91 \times 10^{-7}$ \\
GO:0030335; positive regulation of & 9 & $2.78 \times 10^{-6}$ \\
cell migration & & \\
\hline
\end{tabular}

GO, Gene Ontology.

Table II. KEGG enrichment analyses of differentially expressed genes for epithelia.

\begin{tabular}{lcc}
\hline KEGG term & Count & P-value \\
\hline rno04668; TNF signaling pathway & 8 & $4.01 \times 10^{-6}$ \\
rno04060; cytokine-cytokine receptor & 7 & $1.98 \times 10^{-3}$ \\
interaction & & \\
rno05150; Staphylococcus aureus infection & 4 & $4.39 \times 10^{-3}$ \\
rno04062; chemokine signaling pathway & 6 & $4.47 \times 10^{-3}$ \\
rno04610; complement and coagulation & 4 & $9.76 \times 10^{-3}$
\end{tabular}

cascades

KEGG, Kyoto Encyclopedia of Genes and Genomes; TNF, tumor necrosis factor.

as a significant cluster, which was significantly associated with cytokine-cytokine receptor interaction.

\section{Discussion}

The present study identified the DEGs when comparing the normal, dysplasia and carcinoma expression profiles of tongue cancer samples, which was followed by enrichment analysis and network analysis. For the epithelial samples, 85 DEGs were identified as being significantly differentially expressed among the normal, dysplasia and carcinoma groups, which were associated with neutrophil chemotaxis and inflammatory response GO biological process terms, and associated with the KEGG terms TNF signaling pathway and cytokine-cytokine receptor interaction. In the PPI network, an important module consisting of Cxcl1, Cxcl10, Cxcl13, Cxcl2 and Ppbp was significantly associated with the TNF signaling pathway and cytokine-cytokine receptor interaction. Similarly, for submucosal fibroblast samples, 46 DEGs were identified to be significantly differentially expressed among the three groups, which were associated with neutrophil chemotaxis and inflammatory response GO biological process terms, and with cytokine-cytokine receptor interaction from KEGG analysis. An important module consisting of Illb, Illr2, Illa and Il1rn was significantly associated with cytokine-cytokine receptor interaction.
Table III. GO biological process enrichment analyses of differentially expressed genes for fibroblasts.

\begin{tabular}{lcc}
\hline GO term & Count & P-value \\
\hline GO:0030593; neutrophil chemotaxis & 6 & $3.01 \times 10^{-7}$ \\
$\begin{array}{l}\text { GO:0032496; response to } \\
\text { lipopolysaccharide }\end{array}$ & 8 & $4.97 \times 10^{-6}$ \\
GO:0006954; inflammatory response & 8 & $7.65 \times 10^{-6}$ \\
GO:0019221; cytokine-mediated & 6 & $2.20 \times 10^{-5}$ \\
signaling pathway & & \\
GO:0001660; fever generation & 3 & $8.89 \times 10^{-5}$ \\
\hline
\end{tabular}

GO, Gene Ontology.

Table IV. KEGG enrichment analyses of differentially expressed genes for fibroblasts.

\begin{tabular}{lcc}
\hline KEGG term & Count & P-value \\
\hline $\begin{array}{l}\text { rno04060; cytokine-cytokine receptor } \\
\text { interaction }\end{array}$ & 9 & $4.63 \times 10^{-8}$ \\
rno05323; rheumatoid arthritis & 5 & $8.62 \times 10^{-5}$ \\
rno05146; amoebiasis & 5 & $2.02 \times 10^{-4}$ \\
rno05133; pertussis & 4 & $9.34 \times 10^{-4}$ \\
rno04640; hematopoietic cell lineage & 4 & $1.26 \times 10^{-3}$ \\
\hline
\end{tabular}

KEGG, Kyoto Encyclopedia of Genes and Genomes.

Previous studies regarding bioinformatic analysis of oral tongue cancer have been performed. A methylation profile revealed that hypermethylation of microRNA (miRNA/miR)-10b with downregulation of its target gene nuclear receptor subfamily 4 group A member 3 was associated with tongue tumor patient survival (17). An additional study employed single-nucleotide polymorphism microarrays with human OTSCC samples to indicate that genetic changes, such as 20q11.2 gain encoding the E2F transcription factor 1 gene, may be acquired through clonal evolution and may be responsible for metastasis (18). miR-424 was reported to be a marker specific for tongue tumorigenesis, which also may function in the development of OTSCC (19). However, the majority of studies have focused on the carcinoma itself and ignored other components of the TME.

At present, it is established that the TME has important roles in cancer progression, including primary growth, invasion and metastasis. $(8,9)$ The TME refers to a complex network that primarily consists of cancer cells, fibroblasts, endothelial cells and immune cells, as well as cytokines, growth factors and blood vessels (20). Of these components, cancer-associated fibroblasts have important effects on the formation of the TME and the occurrence of tumorigenesis. In addition, cytokines primarily produced by cancer cells and fibroblasts establish the interaction between cancer cells and other components of the extracellular matrix (21). The importance of both fibroblasts and cytokines has been demonstrated in the current study. According to analysis performed in the 

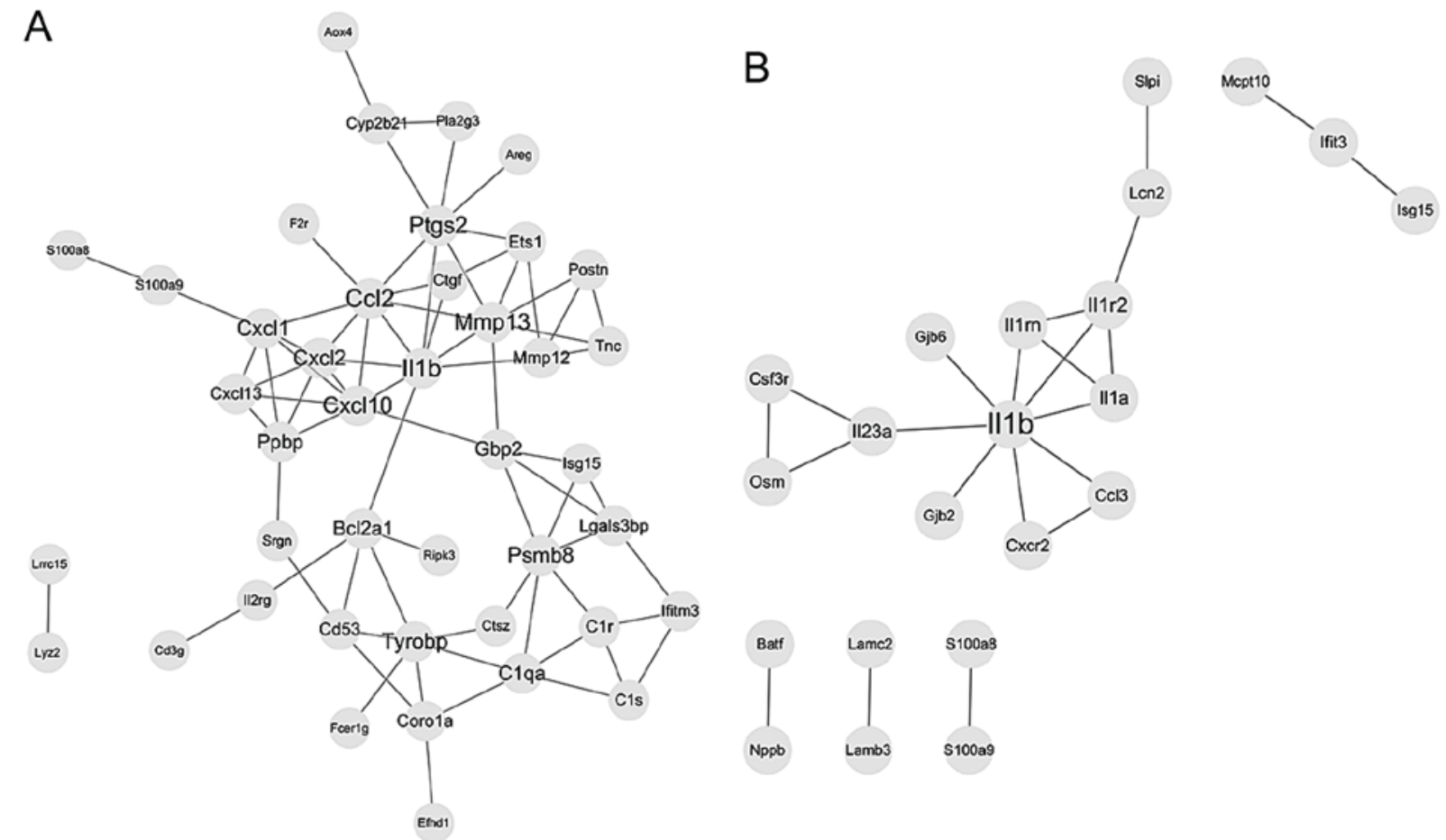

Figure 4. Protein-protein interaction network of differentially expressed genes for (A) epithelia and (B) fibroblasts. The sizes of symbol names indicate nodal connectivity degree.

present study, neutrophil chemotaxis, inflammatory response and cytokine-mediated signaling pathways were enriched in GO analysis of the epithelial and fibroblast samples, while cytokine-cytokine receptor interaction was enriched for KEGG pathways in epithelial and fibroblast samples.

OTSCC is associated with a poor prognosis due to local invasion and lymph node metastases (7). As a result, investigating the potential key functional pathways and the key genes or proteins in the TME is critical for the understanding of the underlying mechanisms of the formation, invasion and metastasis of OTSCC. The results of the present study indicate that the inflammatory immune response and cytokine-mediated signaling interactions have important functions in the tumor microenvironment. Illb was identified as the most important node in both epithelia and fibroblast samples, which was consistent with results obtained by a previous report (13). However, Wu et al (13) only considered FC, while the present study considered FC in addition to the P-value. Wu et al (13) demonstrated that Illb acted as a paracrine signal to activate associated pathways among different cells in the TME, which subsequently established a cycle of reinforcement and generated a comfortable TME for oral carcinogenesis. They subsequently hypothesized that a chemoprevention strategy targeting Illb in the TME may interrupt oral malignant transformation.

The present study discovered a key module that primarily consisted of chemokines, including Cxcl1, Cxcl10, Cxcl13 and $\mathrm{Cxcl} 2$, in epithelia samples, and a key module consisting of Ils, including Il1b, Il1r2, Illa and Il1rn, in fibroblast samples. All of the secreted cytokines may recruit inflammatory cells, which build up in the TME, and the subsequently release of growth factors, cytokines, chemokines and enzymes may regulate tumor growth, angiogenesis, invasion and metastasis (22). The balance between tumor immunity and carcinogenesis may be broken when disequilibrium occurs among secreted factors, which is a prospective antitumor immunity strategy in the future (23).

The current studies aimed to perform gene expression profile analysis of tongue cancer based on a microarray. In addition, the majority of similar published bioinformatic analysis studies were limited to single platforms, including mRNA, miRNA, long non-coding RNA, methylation and mutation. However, a comprehensive global analysis from the Cancer Genome Atlas integrated multi-platform expression characterization from 279 patients with HNSCC. Therefore, studies focusing on integrated multi-platform analysis are essential for the comprehension of mechanisms underlying the TME of tongue tumors (19).

The identification of hub DEGs, enriched pathways and important modules during oral cavity tongue carcinogenesis in TME has the following promising applications: Improve understanding of the potential molecular mechanical and cellular functional changes of tongue malignant transformation of oral cancer; demonstrate demands for integrated multi-platform analysis research regarding the TME; and to reveal the importance of a stable inflammatory immune network in the TME, which is useful for the development of targeted therapy of OTSCC. Additional studies are required to develop a more thorough understanding. The results of the present study may provide insight into the translational practice and future investigation.

\section{Acknowledgements}

The present study was supported by the National Natural Science Foundation of China (grant No. 81272892, 81471352). 


\section{References}

1. Ferlay J, Shin HR, Bray F, Forman D, Mathers C and Parkin DM: Estimates of worldwide burden of cancer in 2008: GLOBOCAN 2008. Int J Cancer 127: 2893-2917, 2010.

2. Blot WJ, Mclaughlin JK, Winn DM, Austin DF, Greenberg RS, Preston-Martin S, Bernstein L, Schoenberg JB, Stemhagen A and Fraumeni JF Jr: Smoking and drinking in relation to oral and pharyngeal cancer. Cancer Res 48: 3282-3287, 1988.

3. Siegel R, Naishadham D and Jemal A: Cancer statistics, 2013 CA Cancer J Clin 63: 11-30, 2013.

4. Castellsagué X, Alemany L, Quer M, Halec G, Quirós B, Tous S, Clavero O, Alòs L, Biegner T, Szafarowski T, et al: HPV involvement in head and neck cancers: Comprehensive assessment of biomarkers in 3680 patients. J Natl Cancer Inst 108: djv403, 2016

5. Greene FL, Page DL, Fleming ID, Fritz A, Balch CM, Haller DG and Morrow M (eds): American Joint Committee on Cancer: AJCC Cancer Staging Manual. 6th edition. Springer, New York, NY, pp157-164, 2002.

6. Rusthoven K, Ballonoff A, Raben D and Chen C: Poor prognosis in patients with stage I and II oral tongue squamous cell carcinoma. Cancer 112: 345-351, 2008

7. Sessions DG, Lenox J, Spector GJ, Chao C and Chaudry OA: Analysis of treatment results for base of tongue cancer. Laryngoscope 113: 1252-1261, 2003.

8. Hanahan D and Coussens LM: Accessories to the crime: Functions of cells recruited to the tumor microenvironment. Cancer Cell 21: 309-322, 2012.

9. Quail DF and Joyce JA: Microenvironmental regulation of tumor progression and metastasis. Nat Med 19: 1423-1437, 2013.

10. Bullinger L, Döhner K, Bair E, Fröhling S, Schlenk RF, Tibshirani R, Döhner H and Pollack JR: Use of gene-expression profiling to identify prognostic subclasses in adult acute myeloid leukemia. N Engl J Med 350: 1605-1616, 2004.

11. Chung CH, Ely K, McGavran L, Varella-Garcia M, Parker J, Parker N, Jarrett C, Carter J, Murphy BA, Netterville J, et al: Increased epidermal growth factor receptor gene copy number is associated with poor prognosis in head and neck squamous cell carcinomas. J Clin Oncol 24: 4170-4176, 2006.

12. Chung CH, Parker JS, Karaca G, Wu J, Funkhouser WK, Moore D, Butterfoss D, Xiang D, Zanation A, Yin X, et al: Molecular classification of head and neck squamous cell carcinomas using patterns of gene expression. Cancer Cell 5: 489-500, 2004.
13. Wu T, Hong Y, Jia L, Wu J, Xia J, Wang J, Hu Q and Cheng B: Modulation of IL-1 $\beta$ reprogrammes the tumor microenvironment to interrupt oral carcinogenesis. Sci Rep 6: 20208, 2016.

14. Ashburner M, Ball CA, Blake JA, Botstein D, Butler H, Cherry JM, Davis AP, Dolinski K, Dwight SS, Eppig JT, et al: Gene ontology: Tool for the unification of biology. the gene ontology consortium. Nat Genet 25: 25-29, 2000.

15. Kanehisa M and Goto S: KEGG: Kyoto encyclopedia of genes and genomes. Nucleic Acids Res 28: 27-30, 2000.

16. Dennis G Jr, Sherman BT, Hosack DA, Yang J, Gao W, Lane HC and Lempicki RA: DAVID: Database for annotation, visualization and integrated discovery. Genome Biol 4: P3, 2003.

17. Krishnan NM, Dhas K, Nair J, Palve V, Bagwan J, Siddappa G, Suresh A, Kekatpure VD, Kuriakose MA and Panda B: A minimal DNA methylation signature in oral tongue squamous cell carcinoma links altered methylation with tumor attributes. Mol Cancer Res 14: 805-819, 2016.

18. Morita T, Uzawa N, Mogushi K, Sumino J, Michikawa C, Takahashi KI, Myo K, Izumo T and Harada K: Characterizing genetic transitions of copy number alterations and allelic imbalances in oral tongue carcinoma metastasis. Genes Chromosomes Cancer 55: 975-986, 2016.

19. Boldrup L, Coates PJ, Laurell G, Wilms T, Fahraeus R and Nylander K: Downregulation of miRNA-424: A sign of field cancerisation in clinically normal tongue adjacent to squamous cell carcinoma. Br J Cancer 112: 1760-1765, 2015.

20. Joyce JA and Pollard JW: Microenvironmental regulation of metastasis. Nat Rev Cancer 9: 239-252, 2009.

21. Polyak K, Haviv I and Campbell IG: Co-evolution of tumor cells and their microenvironment. Trends Genet 25: 30-38, 2009.

22. Lewis CE and Pollard JW: Distinct role of macrophages in different tumor microenvironments. Cancer Res 66: 605-612, 2006.

23. Kortylewski M, Xin H, Kujawski M, Lee H, Liu Y, Harris T, Drake C, Pardoll D and Yu H: Regulation of the IL-23 and IL-12 balance by Stat 3 signaling in the tumor microenvironment. Cancer Cell 15: 114-123, 2009.

This work is licensed under a Creative Commons Attribution-NonCommercial-NoDerivatives 4.0 International (CC BY-NC-ND 4.0) License. 